\title{
ARBEITSRECHTLICHE FRAGEN ZUR TELEARBEIT IN CORONA-ZEITEN
}

von Thomas Luzer

Zusammenfassung: Dieser Beitrag gibt einen kurzen Überblick über die arbeitsrechtliche Situation in Zusammenhang mit Telearbeit. Besonders die aktuellen Gegebenheiten in der Corona-Krise werden kursorisch aufgezeigt. Dabei werden die im Bibliotheksleben auftretenden unterschiedlichen Beschäftigungsformen berücksichtigt.

Schlagwörter: Telearbeit; Homeoffice; COVID-19; Arbeitsrecht; Österreich

\section{LABOUR-LAW RELATED QUESTIONS REGARDING TELEWORK IN THE TIME OF CORONA}

Abstract: This article provides a brief overview of the situation under labour law in connection with teleworking. In particular, the current circumstances surrounding the Corona crisis are outlined in cursory fashion. The different forms of employment occurring in libraries are taken into account.

Keywords: telework; home office, COVID-19; labour law; Austria

DOI: https://doi.org/10.31263/voebm.v73i3-4.5356

(C) Thomas Luzer

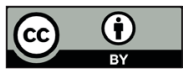

Dieses Werk ist - exkl. einzelner Logos und Abbildungen - lizenziert unter einer Creative-Commons-Lizenz Namensnennung 4.0 International-Lizenz 
Der 16. März 2020 wird uns wohl allen in Erinnerung bleiben. Von einem Tag auf den anderen wurde Österreich zugesperrt. Nach Verordnung der Bundesregierung musste alles, außer den Bereichen, die als lebensnotwendig definiert wurden, zusperren. Das traf natürlich auch auf alle Bibliotheken und die Institutionen, denen diese zugeordnet sind, zu.

Von einem Tag auf den anderen befanden wir uns also im „Home-office“ und verrichteten unsere Arbeit von zu Hause aus vor dem Computer - zumindest den Teil der Arbeit, der auf diese Weise bewältigt werden konnte.

Um eines gleich vorweg zu nehmen: Ich halte „Homeoffice“ für einen völlig ungeeigneten Begriff. Wer verfügt schon zu Hause über ein eigenes Büro? Wie wir den Medien entnehmen konnten, verbrachten viele Menschen diese Tage am Küchentisch und mussten neben ihrer Arbeit noch ihre Kinder unterrichten oder zumindest beim „Homeschooling“ unterstützen.

Ich möchte also hier eine Lanze für die Verwendung des Begriffs „Telearbeit" brechen, da dieser nicht suggeriert, dass wir alle zu Hause ein schön ausgestattetes Büro zur Verfügung haben. Und nicht nur das, dieser Begriff wird auch in den einschlägigen Rechtsvorschriften verwendet.

Wir saßen also zu Hause und arbeiteten so gut es eben ging. Mussten wir das eigentlich? Gibt es eine Verpflichtung zur Telearbeit und wie sind die rechtlichen Rahmenbedingungen?

\section{Allgemeines}

Um diese Frage zu beantworten, muss zunächst einmal dahingehend differenziert werden, dass es in unserem Metier verschiedene Arten der Beschäftigung gibt. In Bibliotheken arbeiten Bundes- und Landesbeamte ebenso wie Vertragsbedienstete. Diese können auch bei ausgegliederten Einrichtungen beschäftigt sein, wo es parallel einen Kollektivvertrag gibt (z. B. Universitäten). Man spricht hier von Alt-VBs. Und dann gibt es noch die sehr große Gruppe derjenigen, die als Angestellte nach „normalem“ Arbeitsrecht beschäftigt sind.

Beginnen wir die Betrachtung zunächst bei den Beamten des Bundes und der Länder. Der Bund hat Telearbeit sowohl im BDG ${ }^{1}$ als auch im $V_{G B}{ }^{2}$ geregelt. Wie bei den meisten Bundesländern normieren diese Regelungen, dass Telearbeit mit den Bediensteten grundsätzlich vereinbart werden kann, wenn es die dienstlichen Obliegenheiten erlauben, der Arbeitserfolg gewahrt bleibt und Geheimhaltungspflichten erfüllt werden können. Die notwendigen Betriebsmittel werden grundsätzlich vom Bund zur Verfügung gestellt. 
Die Regelungen in den Ländern unterscheiden sich dabei oft in wesentlichen Details. So müssen z. B. in der Steiermark keine technischen Geräte zur Verfügung gestellt werden. Im Burgenland kann Telearbeit angeordnet werden, wenn es im öffentlichen Interesse liegt, in Kärnten hingegen nur bei Katastrophenlagen.

Sollten Sie dieser Personengruppe angehören, empfiehlt es sich, mit dem Dienststellenausschuss Kontakt aufzunehmen, falls es zu Streitigkeiten gekommen ist oder solche drohen.

Blicken wir nun auf die große Gruppe der Angestellten. Wie sie den Medien entnehmen konnten, gibt es für diese Personengruppe keine Regelungen, die Telearbeit betreffen. Das bedeutet allerdings nur, dass jegliche Regelungen der Einzelvereinbarung überlassen sind, und somit einen Bestandteil des Arbeitsvertrages darstellen, sofern keine Regelungen im jeweiligen Kollektivvertrag getroffen wurden. Der Kollektivvertrag der Universitäten legt beispielsweise fest, dass über Telearbeit eine schriftliche Vereinbarung zu treffen ist (sonst leider nichts).

Die dritte Gruppe bilden die sogenannten Alt-VBs, also Personen, die früher Vertragsbedienstete waren, nun aber Angestellte ihrer Institution sind. Für diese Gruppe gilt das VBG insofern weiter, als es Bestandteil des Arbeitsvertrags wird. ${ }^{3}$ Für diese Gruppe gilt also das VBG ebenso wie das „normale“ Arbeitsrecht. Antinomien werden mittels des sogenannten Günstigkeitsvergleichs ${ }^{4}$ aufgelöst. Dabei sind aber immer Regelungsgebiete zu vergleichen und keine Einzelregelungen.

Zusammenfassend kann festgehalten werden, dass für die Telearbeit ab 16. März in den meisten Fällen eine Vereinbarung notwendig war. Die notwendige Zustimmung der Arbeitnehmer, egal welchen Typus, ist meiner Meinung nach dadurch gegeben, dass unwidersprochen von zu Hause gearbeitet wurde.

(Die Dienstgeber hätten übrigens auch den Verbrauch von Urlaub vorschreiben können. Bei Beamten und Vertragsbediensteten hätte nur auf maximal zwei Wochen des Urlaubs des Vorjahres zugegriffen werden können, bei Angestellten auf bis zu sechs Wochen Urlaub des Vorjahres und zwei Wochen Urlaub des laufenden Jahres)

Grundsätzlich sollte eine Telearbeitsvereinbarung mindestens Folgendes regeln:

- Gilt Telearbeit generell oder anlassbezogen?

- Wie ist das zeitliche Ausmaß der Telearbeit? Einzelne Tage oder die ganze Woche?

- Regelungen zur Arbeitszeit: Zeitaufzeichnung; Mehrdienstleistungen, Überstunden 
- Eventuelle Erreichbarkeiten

- Art und Umfang der zu leistenden Arbeit

\section{Betriebsmittel und Kostenersatz}

Betriebsmittel sind grundsätzlich vom Dienstgeber bereitzustellen. Da, wo der Arbeitgeber die nötige Ausstattung nicht bereitgestellt hat, stellt sich die Frage nach einem Ersatz. Anschaffungen, die ich tätigen musste, um meine Telearbeit verrichten zu können, sind dann zu ersetzen, wenn diese neu auf mich hinzugekommen sind. (Beispiel: Ich wurde verpflichtet, an Videokonferenzen teilzunehmen. Dazu musste ich mir ein Headset kaufen, diese Kosten sind zu ersetzen, wobei ein Teil für Privatnutzung abgezogen werden kann). Aufwandsersatz stellt hingegen nach allgemeinen zivilrechtlichen Regeln eine dispositive Größe dar (Beispiel: Ich habe eine tolle technische Ausstattung und bringe diese in meine Arbeit ein; einen Ersatz für die Abnutzung muss ich ebenso ausverhandeln wie eine Beteiligung an der Stromrechnung etc.).

\section{Arbeitszeit}

Die Arbeitszeit sollte vom Dienstgeber möglichst klar definiert werden. Dies ist vor allem in Hinblick auf die zwingenden Arbeitsruhebestimmungen relevant. Eine gewisse Erleichterung könnte hier die Regelung des $\S 26$ Abs. 3 AZG darstellen: „Für Arbeitnehmerinnen/Arbeitnehmer, die die Lage ihrer Arbeitszeit und ihren Arbeitsort weitgehend selbst bestimmen können oder ihre Tätigkeit überwiegend in ihrer Wohnung ausüben, sind ausschließlich Aufzeichnungen über die Dauer der Tagesarbeitszeit zu führen.“

Die eigentliche Idee hinter dieser Regelung war freilich nicht Corona, sondern die Zeitaufzeichnung bei Dienstreisen. Ob sie in Corona-Zeiten eine praktikable Möglichkeit darstellt, wird sich erst weisen. Die aktuelle Judikatur des EUGH spricht eher dagegen, da das Gericht eine klare Verpflichtung des Arbeitgebers bei der Aufzeichnung von Arbeitszeiten sieht. ${ }^{5}$

\section{ArbeitnehmerInnenschutzgesetz}

Das eigene Wohnzimmer wird nach herrschender Ansicht nicht als Arbeitsstätte im Sinne des ASchG betrachtet. Es wäre ja auch wahrlich nicht 
erfreulich, wenn das Arbeitsinspektorat unaufgefordert Zutritt zu meinen vier Wänden hätte.

Daraus kann aber nicht abgeleitet werden, dass Arbeitnehmerschutz für den Arbeitgeber kein Thema mehr ist, wenn die Arbeit von zu Hause aus erledigt wird. Aus den $\S \S 3$ u. 4 ASchG ergibt sich vielmehr eine Verpflichtung des Arbeitgebers, sich generell um die Gesundheit der Bediensteten zu sorgen. Dieser Verpflichtung kann bei Telearbeit beispielsweise durch Verhaltensrichtlinien und ähnlichem entsprochen werden.

\section{Steuerrecht}

Eine steuerliche Berücksichtigung der Mehrkosten, die durch Telearbeit entstehen, ist im österreichischen Steuerrecht derzeit nicht verankert. In Deutschland wurde bereits ein Betrag von EUR 5.- pro Tag in Telearbeit festgelegt, der bei der Steuererklärung geltend gemacht werden kann.

\section{Ausblick}

Die Sozialpartner verhandeln derzeit intensiv über eine gesetzliche Regelung zur Telearbeit, die möglichst alle rechtlichen Aspekte abdecken soll. Das Problem dabei ist freilich, dass Telearbeit nicht gleich Telearbeit ist. Zu hoffen bleibt, dass die Regelung möglichst flexibel ist und möglichst viel der Vereinbarung zwischen Arbeitgeber und Arbeitnehmer überlässt. Ich finde Telearbeit ein bis zwei Tage pro Woche sehr angenehm und verzichte dabei gerne auf einen pauschalierten Ersatz von Stromkosten. Umgekehrt darf aber auch niemand gezwungen werden (außer in einem „Lockdown“) von zu Hause zu arbeiten. Dafür hat Arbeit an sich viel zu viele soziale Aspekte.

Auch eine Novelle des Arbeitszeitgesetzes würde ich begrüßen. Gerade die Zeit, in der alle zu Hause waren, hat gezeigt, dass das sture Festhalten an Arbeitszeiten und deren Aufzeichnung überholt ist. Im Mittelpunkt sollte vielmehr die Leistungserbringung stehen.

Vieles wird auch von noch zu verhandelnden Betriebsvereinbarungen abhängen, da zu erwarten ist, dass der Gesetzgeber hier Ermächtigungen erteilen wird.

Fortsetzung folgt, wenn der Bundesgesetzgeber neue Regeln erlässt. 
Dr. Thomas Luzer Universität Wien, Fachbereichsbibliothek Rechtswissenschaften E-Mail: thomas.luzer@univie.ac.at

\section{Literatur}

Bundesgesetz vom 27. Juni 1979 über das Dienstrecht der Beamten (Beamten-Dienstrechtsgesetz 1979 - BDG 1979). https://www.ris.bka. gv.at/GeltendeFassung.wxe?Abfrage=Bundesnormen\&Gesetzesnumm er $=10008470$

Bundesgesetz vom 17. März 1948 über das Dienst- und Besoldungsrecht der Vertragsbediensteten des Bundes (Vertragsbedienstetengesetz 1948 - VBG). https://www.ris.bka.gv.at/GeltendeFassung.wxe?Abfrage=Bun desnormen\&Gesetzesnummer $=10008115$

Bundesgesetz über die Organisation der Universitäten und ihre Studien (Universitätsgesetz 2002 - UG). https://www.ris.bka.gv.at/GeltendeFassung.wxe? Abfrage=Bundesnormen \&Gesetzesnummer $=20002128$

Bundesgesetz vom 14. Dezember 1973 betreffend die Arbeitsverfassung (Arbeitsverfassungsgesetz - ArbVG). https://www.ris.bka.gv.at/GeltendeFassung.wxe? Abfrage=Bundesnormen\&Gesetzesnummer=10008329 Urteil vom 14.05.2019, Federación de Servicios de Comisiones Obreras (CCOO)/Deutsche Bank SAE, C-55/18, ECLI:EU:C:2019:402. http:// curia.europa.eu/juris/liste.jsf?language $=$ de\&num $=\mathrm{C}-55 \% 2 \mathrm{~F} 18$

1 BDG § 36a.

2 VBG $\S 5 c$.

3 Siehe dazu beispielsweise UG 2002 § 126 (4).

4 Siehe dazu ArbVG § 3.

5 EUGH vom 14.5.2019 C55/18. 